\title{
A preliminary study of beat-to-beat blood pressure biofeedback using the vascular unloading method
}

\author{
YUKIHIRO SAWADA ${ }^{1}$ \\ Department of Human Science, Sapporo Gaknin Universily, Ebetsu, Hokkaido 069-01 \\ KEN-ICHI YAMAKOSHI \\ Research Institute of Applied Electricity, Hokkaido University, Kita-ku, Sapporo 060
}

\begin{abstract}
Eight male normotensive, undergraduate students participated as subjects in blood pressure (BP) biofeedback training of two, 12-min periods. BP was measured continuously using the vascular unloading method (Yamakoshi, Shimazu, \& Togawa, 1980), and the BP information was fed back to the subject on a beat-to-beat basis. They were trained to control either their systolic or diastolic BP in both upper and lower directions. Training was successful in five subjects for BP raising ( 3 for systolic and 2 for diastolic) and in one subject for BP lowering (diastolic). Although it is too early to draw a firm conclusion at present, from one simple experiment, the vascular unloading method appears to be feasible to BP biofeedback researchers in future studies. A possible mechanism of target BP changes, in addition, is discussed based on some physiological data obtained from simultancous recordings. The possibility of applying a nonparametric randomization test for a single subject to the present data is also considered.
\end{abstract}

Key words: blood pressure, the vascular unloading method, biofeedback, single-case experimental design.

Noninvasive and continuous blood pressure (BP) measurement for a long period of time without much discomfort is a prerequisite for a BP biofeedback experiment. Some methodological advances have been made recently to overcome these problems (Elder, Longacre, Welsh, \& McAfee, 1977; Lee, Caldwell, \& Lee, 1977; Shapiro, Greenstadt, Lane, \& Rubinstein, 1981; Turskey, Shapiro, \& Schwartz, 1972). However, constraints have persisted even after these improvements, and partly for that reason, $\mathrm{BP}$ biofeedback studies have been carried out infrequently.

The vascular unloading method, developed by Yamakoshi, Shimazu, and Togawa (1980), seems to solve the aforementioned problems (Sawada, Yamakoshi, \& Shimazu, 1983). The present

1 Address all correspondence to Yukihiro Sawada, Department of Human Science, Sapporo Gakuin University, 11 Bunkyodai, Ebetsu, Hokkaido 069-01. study is a preliminary report of a BP biofeedback experiment using this method to illustrate its applicability in biofeedback research.

\section{Method}

\section{Subjects}

The subjects were eight male normotensive, undergraduate students varying in age from 20 to 23 . They have been involved in extracurricular drama activities for three years or more. Such students were intentionally selected as subjects since it was thought that they could provoke vivid images more easily than other students. They were paid a small fixed sum of money irrespective of performance.

\section{Measurement}

Beat-to-beat systolic and diastolic BP (SBP and DBP) were measured using the vascular unloading method (USM-801; 
Ueda, Tokyo, Japan). This instrument has been described in detail elsewhere (Sawada et al., 1983; Iamakoshi et al., 1980). In Brief, the root portion of the left middle finger is enclosed in a thin, water-filled cuff. Changes in vascular volume associated with intra-arterial pressure are detected by a photoplethysmograph, and are compensated by counter pressure from the cuff by use of a hydraulic servosystem in order to maintain the artery in an unloaded state (zero transmural pressure). In this fashion, the cuff pressure instantaneously tracks intra-arterial BP.

Simultaneous recordings were made of the stroke volume (SV) and cardiac output (CO) using a transthoracic admittance plethysmograph (Ito, Yamakoshi, and Togawa, 1976) and heart rate (HR) through a lead II electrocardiogram configuration. Total peripheral resistance (TPR) was calculated from the $\mathrm{CO}$ and mean BP values. These SV, HR, CO, \& TPR are the intervening cardiovascular parameters through which $B P$ biofeedback is thought to influence the target $\mathrm{BP}$ responses.

In addition, electromyogram was measured using surface electrodes from the left forearm extensor and was processed through an integrator to evaluate the degree of muscle tension (MT), Respiration was recorded using a straingauge transducer mounted on a belt placed around the chest, and the respiratory rate (RR) was obtained. These MT and RR are considered to constitute important parts of the somatic mediational parameters through which BP biofeedback affects the intervening cardiovascular activities above-mentioned.

Among the output signals, electrocardiogram, respiration, and integrated electromyogram were recorded on a San-ei 140 polygraph (Nippon Electronic Company). The BP waveforms were subjected to an on-line computer analysis (PC9801F personal computer, Nippon
Electronic Company) in order to detect the SBP and DBP values, as well as to present the beat-to-beat BP information on a computer display.

\section{Procedure}

Each subject underwent a three min adaptation, six 1 min biofeedback training sessions interspersed by six 30 s baseline sessions (the first half of the experimental period), a three min rest, and another six pairs of baseline-training sessions (the second half of the experimental period) in a sound-proofed shield room. According to the types of target $\mathrm{BP}$ responses (SBP, DBP) and the directions of $\mathrm{BP}$ controls (DOWN, UP), every two subjects were allocated to one of the following training patterns from the first to the second half of the experimental period: SBP/DOWN-UP, SBP/UP-DOWN, DBP/ DOWN-UP, DBP/UP-DOWN.

At the beginning of each training session, the mean of target $B P$ values in the preceding baseline session was preset as the criterion for the control. Then, on the computer display, the difference of target $\mathrm{BP}$ response from the preset mean value was plotted successively against a graph frame, in which a horizontal line corresponded to the criterion. The plotted continuous data path was renewed every 10 beats throughout the training session. Before starting each half of the experimental period, the subject was advised to utilize any kind of mental image available for controlling his target BP. However, he was reminded to maintain a normal breathing pattern and not to intentionally change muscle tension during the BP control.

\section{Statistical Analysis}

The nonparametric randomization (RD) test for a single subject was used for a statistical evaluation of the biofeedback effects (Levin, Marascuilo, \& Hubert, 1978). The mean values of SBP \& $\mathrm{DBP}$, as well as those of $\mathrm{SV}, \mathrm{HR}, \mathrm{CO}$, 
and TPR during each baseline and training session were calculated as the summary statistics that may lead to approximatcly independent, or at least, uncorrelated random variables because they were obtained from overall within-session observations. For the RR and MT values, they were regarded as summary statistics in themselves. The results of the first baseline and training sessions were discarded.

In the RD test, the observed data set is considered to bc a random sample of all the possible assingnments to the conditions. In the present experiment, therefore, there are $252\left(={ }_{10} C_{5}\right)$ possible data subsets that can be assigned to one of the conditions (e.g., biofeedback training) in each half of the experimental period for each physiological activity under the null hypothesis. The sum of 5 summary values was calculated for each of a possible 252 assignments and, based on these sums, the probability distribution was defined. Probabilities of the sums which are equal to or more extreme than the actual sum for the given result were totalled, and the doubling of this total was compared with the standard significance levels.

\section{Results}

Figure 1 shows an example of the representative results. The upper half is the results of subject-3 (SBP/UP-DOWN), and the lower half is those of subject-5 (DBP/DOWN-UP). Mean SBP and DBP values for the baseline (B: unfilled circle) and training ( $\mathrm{T}$ : filled circle) sessions are plotted and are connected with bold lines in case of the target BP responses. As shown in Fig. 1, subject-3 was very good at controlling his target $\mathrm{BP}$ (SBP) in the upper direction during the first half of the experimental period. The non-target BP (DBP) changed correspondingly. The same subject, however, failed to lower his SBP in the second half. Subject-5, on the other hand, controlled his target BP (DBP) successfully downward to some degree, but during the second biofeedback training, he could not elevate his DBP anymore.

From the RD test, it was shown that six out of eight subjects controlled their target BP's successfully; and that five out of the six successful subjects were skillful in raising their target BP's between six and $15 \mathrm{mmHg}$ (three for SBP and two for DBP), while only one subject could achieve his target DBP lowering of about $6 \mathrm{mmHg}$. It was also intriguing that none of the six subjects could both raise and lower his target BP significantly. Furthermore, the five subjects successfully trained to raise their BP's achieved good control of their target BP's in the first half of the experimental period. It is noted, however, that this superiority might be attributed not to the order of the experimental period but to the direction of target BP control since one subject who had additional biofeedback training demonstrated a significant raise in target $\mathrm{BP}$ in the second half of this new experiment.

In five of six successful subjects, changes in the target BP's were mostly associated more with those in the HR's and CO's than in the SV's and TPR's. Concerning the RR's and MT's, corresponding changes were shown in the former for all of the six successful subjects but not shown in the latter.

The results of an open ended questionnaire filled out after the experiment suggested that every subject had tried to actively utilize certain available images during the target BP raising. However, during the BP lowering, half of the subjects did not try to elicit any particular images, but rather sought to erase provoking images. This was true even in subject-5 who was the only person successful in lowering his target BP (see Fig. 1). 


\section{Discussion}

In the present experiment, the vascular unloading instrument worked quite well so that failures in BP monitoring due to artifact or faulty instrument movement rarely occurred. None of the subjects complained of discomfort or pain during the BP measurements which lasted 12 min during each half of the experimental period. The SBP and DBP values for the same cardiac cycle were obtained accurately on a beat-to-beat basis, and the target BP information was continuously and uninterruptedly displayed as feedback signals. The applicability of this method in BP biofeedback experiment was thus illustrated.

Although the results may not have such important implications from a clinical standpoint because BP raising (but not lowering) controls were much easier for the present subjects, the results are helpful for future studies in that they shed light on a possible mechanism of $\mathrm{BP}$ raising control through biofeedback training. From the changes shown in the mediational as well as in the intervening cardiovascular parameters during the BP raising training, the following process may be assumed: first, RR becomes elevated through active utilization of available images; second, increments in HR

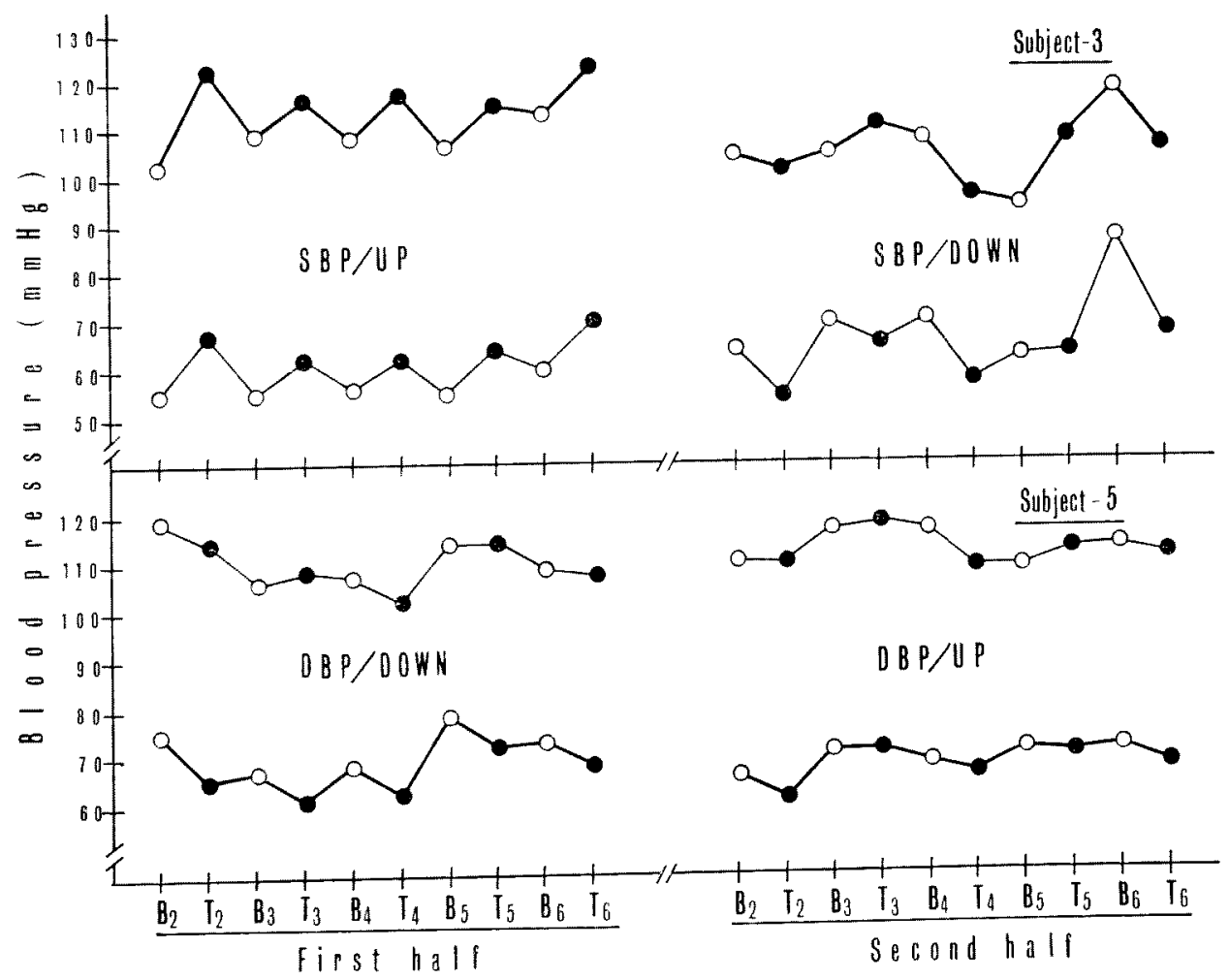

Fig. 1. An example of the representative results. The means of systolic and diastolic blood pressurc (SBP and DBP) under the baseline (B: unfilled circle) and training ( $T$ : fillcd circle) sessions for the first and second half of the experimental period in subject-3 and subject-5 are plotted. Their training patterns are SBP/UP-DOWN and DBP/DOWN-UP respectively. Target BP responses are connected with bold lines. BP responses in the first baseline and training sessions are excluded. 
and $\mathrm{CO}$ are produced by the higher $\mathrm{RR}$; and thirdly, target $\mathrm{BP}$ raising is attained as the result of changes in these cardiovascular parameters. In contrast, BP lowering appears to be difficult because the subjects were not helped by the appropriate images available for producing $\mathrm{R} R$ reduction.

This mediation of BP by respiration is not unexpected, considering the profound influences of respiration on cardiovascular parameters (Grossman, 1983). Nevertheless, it is plausible that successive beat-tobeat feedback of the BP information, which may be called a "time-series" feedback, enabled the subjects to recognize the natural relationship between $\mathrm{BP}$ variations and breathing patterns much more easily. For the intervening cardiovascular parameters, it may be noted that short term BP control in an upward or downward direction can be accomplished predominantly not through changes in TPR but through those in $\mathrm{CO}$ and HR. Nearly the same results were reported by Messerli, Decarvalho, Christie, and Frohlich (1979) who investigated the hacmodynamic effects of BP biofeedback in borderline hypertensives.

Application of single-case experimental design was of an additional interest in the present study. Several distinct advantages of single-case design for biofeedback research have been suggested (Barlow, Blanchard, Hayes, \& Epstein, 1977). Large individual differences in physiological activities and in the acquisition of control ability commonly observed in biofeedback training can be suitably appraised in single-case designs. The present application illustrated that the RD test could be useful in biofeedback research if the data are summarized into treatment phases and predictions are made about the ordering of outcomes.

The present study, as a whole, suggests the availability of the vascular unloading method in future BP biofeedback experiments. However, much research is needed to draw a firm conclusion about the value of $\mathrm{BP}$ training using this method because of too small a sample size and too short training periods in the present study.

\section{References}

Barlow, D. H., Blanchard, E. B., Hayes, S. C., \& Epstein, I. H. 1977 Single-case designs and clinical biofeedback experimentation. Biofeedback and Self-Regulation, 2, 221-239.

Elder, S. T., Longacre, A., Jr., Welsh, D. M., \& McAfee, R. D. 1977 Apparatus and procedure for training subjects to control their blood pressure. Psychophysiology, 14, 68-72.

Grossman, P. 1983 Respiration, stress, and cardiovascular function. Psychophysiology, 20, 284300.

Ito, H., Yamakoshi, K., \& Togawa, T. 1976 Transthoracic admittance plethysmograph for measuring cardiac output. Journal of Applied Physiology, 40, 45l-454.

Lee, R. M., Caldwell, J. R., \& Lee, J. A. 1977 Blood pressure tracking systems and their application to biofeedback. Biofeedback and SelfRegulation, 2, 435-447.

Levin, J. R., Marascuilo, L. A., \& Hubert, L. J. $1978 \mathrm{~N}=$ nonparametric randomization tests. In T. R. Kratochwill (Ed.), Single subject research: Strategies for evaluating changes. New York: Academic Press. Pp. 167-196.

Messerli, F. H., Decarvalho, J.G.R., Christie, B., \& Frohlich, E. D. 1979 Systemic haemodynamic effects of biofeedback in borderline hypertension. Clinical Science, 57, 437-439.

Sawada, Y., Yamakoshi, K., \& Shimazu, H. 1983 Vascular unloading method for noninvasive measurement of instantaneous arterial pressure: Applicability in psychophysiological research. Psychophysiology, 20, 709-714.

Shapiro, D., Greenstadt, L., Lane, J. D., \& Rubinstein, E. 1981 Tracking-cuff system for beat-to-beat recording of blood pressure. Psychophysiology, 18, 129-136.

Turskey, B., Shapiro, D., \& Schwartz, G. E. 1972 Automated constant cuff-pressure system to measure average systolic and diastolic blood pressure in man. IEEE Transactions on Bio-medical Engineering, BME-19, 271-276.

Yamakoshi, K., Shimazu, H., \& Togawa, T. 1980 Indirect measurement of instantaneous arterial blood pressure in the human finger by the vascu- 
lar unloading technique. IEEE Transactions on Bio-medical Enginering, BME-27, 150-155.
(Received Oct. 21, 1989; accepted July 14, 1990) 